\section{Blood-brain barrier}

Blood-Brain Barrier in Physiology and Medicine. By Stanley I. Rapoport. Pp. xii+316. (Raven: New York, 1976.) \$28.

THE concept of the blood-brain barrier derives from the classical studies on intravital staining carried out by Ehrlich and Goldmann early in this century; they observed that parenteral injections of acidic dyes, such as Trypan blue, resulted in staining of connective tissues but not of central nervous tissue. Until the mid1950 s the concept remained rather vague and its reality was often called into question, in spite of several quantitative studies, mainly from Brodie's laboratory.

In 1956 the reviewer published his Physiology of the Ocular and Cerebrospinal Fluids, a book in which the dynamic aspects of exchanges within the central nervous system were related to current concepts of transport in biological systems; ten years later his Physiology of the Cerebrospinal Fluid integrated the considerable accession of knowledge that probably resulted from the stimulus to research in the field of brain transport provided by his first monograph. More than ten years have elapsed since the publication of this latter book, and during this period not only has research been proceeding rapidly in the field of brain transport but also several monographs and symposium volumes have been published that have amplified and brought up to date many of the aspects covered by the Physiology of the Cerebrospinal Fluid.

Rapoport's Blood-Brain Barrier may be looked at in this context; the basic ideology and many of the experimental facts were already familiar before he began to write the book, and it is interesting to consider how he has approached his task and with what success. After a brief introduction to cell structure, the second chapter presents, in lucid fashion, the basic elements of transport across cell membranes, proceeding from the sample case of a single cell to the more complex one of transport across epithelial layers. In the next chapter quantitative aspects of the barrier between blood and extracellular space of the brain are dealt with, and the site of the barrier is, in accordance with recent studies of Reese and Karnovsky, definitely attributed to the capillary endothelium, which differs from that of connective tissue in having its intercellular clefts sealed by tight junctions.

In the next chapter the quantitative aspects of brain capillary permeability are analysed in the light of existing information; this is an especially valuable chapter, in which the author describes his own recent studies on the "opening of the barrier' by preliminary treatment of brain tissue with hypertonic solutions, a treatment that reversibly seems to open up intercellular junctions that had been tight. The chapter also includes a discussion on brain oedema, a discussion that, in the reviewer's opinion, betrays a defective understanding of the importance of solute flow across the barrier. In connective tissue, oedema follows from a reduction of the colloid osmotic pressure of the plasma or from an increase in that in the extracellular spaces, in accordance with the Starling principle. The flow of fluid in response to altered colloid osmotic relations is feasible because of the absence of significant restraints on the passage of the non-colloidal dissolved constituents of plasma. In the brain, however, this is not true, so that a mere alteration in colloid distribution can have little effect on the water distribution between blood and tissue.

Two chapters deal with pathology of the barrier and the regulation of drug entry; this latter seems unnecessary as a

\section{Colourful chemistry}

Colour and Constitution of Organic Molecules. By J. Griffiths. Pp. ix +281. (Academic: London and New York, 1976.) $£ 9.50$; $\$ 20.75$.

To a colour chemist the absorption spectra of organic molecules between wavelengths of 400 and $700 \mathrm{~nm}$ is a matter of overriding importance. To an organic chemist it is a relatively narrow part of the whole spectral range in which electronic transitions can be detected. Technologically, dyestuffs have important properties that distinguish them as a class amongst organic molecules, but the principles underlying the interpretation of their spectra are the same as for any colourless organic molecule that absorbs in the ultraviolet region of the spectrum.

In this book, John Griffiths provides a brief but useful review of the molecular orbital method at both the Huckel and semi-empirical SCF level. These theories, in so far as they are used to interpret organic electronic spectra, are a product of the 1950s. The all-electron methods developed later have had little impact on the subject, perhaps because of the success of these earlier theories. The book also contains a brief account of the theory of band intensities, lifetimes and the Franck-Condon envelope.

The crux of the book, its raison d'être, is a suggested classification of coloured organic compounds into "four broad classes". These are $n \rightarrow \pi^{*}$ chromogens, donor-acceptor chromo- separate chapter since the facts belong to the general subject of barrier permeability. The same may be said of the following chapter, which deals with transport of sugars, amino acids and "other substances'. The chapter is valuable in summarising our knowledge but should have followed immediately on the earlier description of kinetics.

In the final chapter the author follows the reviewer's precedent and brings the barriers in the eye in relation to those in the brain; especially interesting are the phenomena of 'osmotic opening' of the barriers in the eye.

Rapoport has provided a very useful book that gives a clear view of the basic phenomena and their interpretation to the uninitiated, and provides a summary of recent work that is valuable to the established worker in the field.

Hugh Davson

Hugh Davson is Honorary Research Fellow at King's College, University of London, UK.

gens, cyanine-type chromogens, and acyclic and cyclicpolyene chromogens. The last class is rather broad and is discussed under four sub-classes, acyclic polyenes, polycyclic benzenoid compounds, non-benzenoid alternant cyclic systems and non-alternant systems. This type of classification may be new to colour chemists but it closely follows the pattern of chapters in several books of organic spectroscopy.

The part of the book which comes closest to providing some analysis of organic electronic spectra which is not found in other, more comprehensive, texts is that which deals with donoracceptor chromogens. The importance of this part of the book can be seen by the statement: "with the exception of the polycyclic quinones and the phthalocynanines, all the commercially important synthetic dyes are of this type". This is a good account with reference to several important papers. I was, however, surprised to see no acknowledgment to the work on interand intramolecular charge-transfer spectra of Mulliken, Nagakura and others. The absence of an author index prevented me from checking that this acknowledgment was not hidden in some other part of the book.

John Griffiths has written a useful book. I do not believe that it contains much that will be new to those whose interest has been the wider subject of the electronic spectra of organic compounds but it may encourage more colour chemists to discover the theoretical advances that have been made in this field.

\section{J. N. Murrell}

J. N. Murrell is a Professor of Chemistry at the University of Sussex, UK. 